\title{
A Case Report of an Acute Confusional State, in an Elderly Female Patient with Venous Thrombosis of the Leg
}

\author{
Ahmed Al-Imam ${ }^{1,2,}$ \\ ${ }^{1}$ Novel Psychoactive Substances Research Unit, Department of Postgraduate Medicine, School of Life and Medical Sciences, Hertfordshire University, United Kingdom \\ ${ }^{2}$ Department of Anatomy and Cellular Biology, Faculty of Medicine, University of Baghdad, Iraq \\ "Corresponding author: Ahmed Al-Imam, House 18/5, Al-Akhtal Street, District 318, Al-Adhamyia, 10053, Baghdad, IRAQ. Tel/Fax: +964-7714338199, E-mail: tesla1452@gmail.com
}

Received 2016 March 19; Revised 2016 August 20; Accepted 2016 October 07.

\begin{abstract}
Introduction: Delirium, also known as acute Confusional state, is a common presentation in geriatrics. It is characterized by an acute and a fluctuating disturbance of consciousness, and a deficit in cognitive functions. Delirium that occurs in demented people is referred to as delirium superimposed on dementia.

Case Presentation: The patient presented here was a challenging case of an elderly female with 86 years of age that was diagnosed with delirium superimposed on dementia (DSD). The patient developed DSD in parallel with venous thrombosis (VT) affecting her right leg. Venous thrombosis was initially superficial, affecting the great saphenous vein (GSV), which later progressed to deep venous thrombosis (DVT). The therapeutic approach for this patient was literally a dilemma, and a distressing situation to the patient, medical and paramedical staff, and the caregivers. The patient refused feeding, and had refractory insomnia. Additionally, the patient became frequently hyperactive throughout the day in association with frequent delirium attacks. The hyperactivity was difficult to control, despite her leg condition (DVT) that required cautious immobilization to prevent the catastrophe of a superimposed episode(s) of thromboembolic phenomenon. Luckily, a pulmonary thromboembolism never happened.

Conclusions: The patient resisted, and sometimes completely rejected diagnostic or therapeutic intervention, and medical consultations. A multidisciplinary approach was mandatory, and the DSD was eventually successfully well-managed, with an adjusted dose of haloperidol, a high potency first generation antipsychotic drug. Subsequently, her medical and surgical complications were also successfully managed. Finally, the patient was discharged from the hospital, and later scheduled, for a reconstructive surgery, using autologous mesh skin grafting under regional anesthesia, to cure her refractory venous leg ulcer. The venous ulcer occurred as a complication for the venous thrombosis.
\end{abstract}

Keywords: Delirium, Dementia, Haloperidol, Heparin, Varicose Ulcer, Venous Thrombosis

\section{Introduction}

Delirium, also known as acute confusional state, is a common geriatric syndrome, and occasionally presents as a medical emergency. It is characterized by an acute and a fluctuating disturbance of consciousness, inattention and deficits in arousal and cognitive functions. Delirium that occurs in demented individuals is known as delirium superimposed on dementia (DSD). The prevalence of DSD in the community ranges from $22 \%$ to $89 \%$. It is associated with serious adverse outcomes including an accelerated decline in cognitive functions, hospitalization and re-hospitalization, and high mortality rates. Furthermore, DSD might be a sign of an undiagnosed infection or a pharmacologically-induced phenomenon. The diagnosis of DSD is often challenging, because signs of delirium are usually fluctuating even during the same day, and might be confused with the underlying dementia, and the abnormal cognitive status. cognitive functions in DSD can be assessed by: confusion assessment method (CAM), CAM for the Intensive Care Unit (CAM-ICU), cognitive test for delirium (CTD), delirium rating scale (DRS), electroencephalography (EEG), and short-portable mental status questionnaire (SPMSQ). In light of the current medical evidence, the CAM and the CAM-ICU represent the best tools, to be used for evaluating and confirming the diagnosis of DSD. However, caregiver information is also vital, to ascertain whether there has been an acute decline of cognition as a characteristic of delirium, or a much longer decline as a characteristic of dementia $(1,2)$.

The diagnosis of the underlying dementia in this case scenario, was based on a conclusive diagnostic criterion by McKhann et al., 2011, at the National Institute on AgingAlzheimer's association workgroups $(2,3)$. This was a case

Copyright @ 2016, Mazandaran University of Medical Sciences. This is an open-access article distributed under the terms of the Creative Common 
of DSD in an elderly female patient (86 years old), whom initially presented superficial venous thrombosis (SVT) affecting the right lower limb, specifically her right leg, that later progressed to deep venous thrombosis (DVT). Luckily, the patient DVT, did not progress into pulmonary embolism (PE). However, it is known that SVT is closely linked to deep vein thrombosis (DVT) or pulmonary embolism (PE), which highlights the potential severity of this disease. Deep Vein Thrombosis or PE is diagnosed in 20\% - 30\% of SVT $(4,5)$. The patient was already diagnosed with dementia, and an acute confusional state was later superimposed on her, that evolved during the course of her illness, to form this intriguing case of DSD, for which an MDT approach was required.

\section{Case Presentation}

The patient was an 86-year-old female from Baghdad, Iraq. She was a non-diabetic. She was a chronic hypertensive patient for over five decades. Her blood pressure was well-controlled with an anti-hypertensive medication, Lisoretic (Lisinopril and Hydrochlorothiazide) $10 \mathrm{mg} / 12.5$ mg tablet, once daily. She had chronic bilateral dependent leg edema for over 30 years, for which graded compressive elastic hosiery was used. In the past two years, the patient developed two episodes of superficial thrombophlebitis (SVT), affecting the veins of her right leg, specifically the great saphenous vein (GSV). Patient recovered successfully from both episodes without any complications. The patient was successfully treated with a conservative therapeutic medical protocol.

On the 18th of October 2015, she developed the 3rd episode of SVT, affecting the veins of the right lower limb, specifically the GSV. Initially, the inflammation affected the vein at a distance of four centimeters $(\mathrm{cm})$ above the medial malleolus, for a longitudinal venous segment of approximately six centimeters in length. Later, within two days, the inflammation spread to affect the crural segment of the GSV and the overlying skin, starting from the level the medial malleolus to the level of medial condyle of tibia. However, the patient was systemically well, afebrile and vitally stable. Clinically and biochemically, cellulitis was excluded. However, the patient was unable to walk normally due to some degree of pain. The overlying skin was moderately red, tender, with a tender cord-like vein on palpation. The patient was referred to a vascular surgery clinic, and the diagnosis of chronic venous insufficiency leading to SVT, was confirmed. Management was initiated, using daily Lincomycin intramuscular injections, Hirudoid (Heparinoid) gel application twice daily, Daflon $500 \mathrm{mg}$ tablet once daily, $100 \mathrm{mg}$ Celecoxib tablet twice a day, and warm baths for the affected leg.
Within a few days of initiating this management, the patient showed dramatic improvement, and the visiblepalpable inflammation regressed significantly. However, the edema of the right leg persisted and was most evident at the foot; there was also a residual hard area located about four centimeters above the level of the medial malleolus. The hard area was mildly tender, but with normal skin color. The patient was encouraged to stay mobile, and to do daily warm baths for the affected leg. Reparil gel, applied twice daily, was later added. However, no significant improvement was noticeable. Later, the hardened area evolved a slightly depressed centrally-located brownblack dyspigmentation, approximately two millimeters in diameter, that slowly progressed over two months, into a wide necrotic area of skin, with clinical characteristics of a developing venous leg ulcer.

Doppler study of both lower limbs was done, in parallel with laboratory investigations including D-dimer level assessment. Douma et al. proposed the age-adjusted D-dimer cut-off value. This cut-off value of D-dimer level, increases the number of older patients in whom deep vein thrombosis can be safely excluded. The cut-off value is calculated by multiplying the patient's age by 10 in patients older than 50 years old (6). Unfortunately, this particular patient was not excluded from DVT, her D-dimer level was $876.27 \mathrm{ng} / \mathrm{mL}$, while the adjusted cut-off value is $860 \mathrm{ng} / \mathrm{mL}$. Furthermore, Doppler studies confirmed the presence of thrombosis affecting the perforating veins between the GSV and deep veins (venae comitantes of tibial arteries).

Collectively, based on patient's clinical features, Doppler studies, and the level of D-dimer adjusted for age, the diagnosis of deep venous thrombosis (DVT) of the right leg, was confirmed. The patient also had serially elevated renal function tests (RFT) while urea levels were fluctuating in the range of $80 \mathrm{~s}-100 \mathrm{~s} \mathrm{mg} / \mathrm{dl}$, with an elevated creatinine level up to $2.1 \mathrm{mg} / \mathrm{dL}$, while serum electrolytes were completely normal. At this point in time, the patient started to develop features of an acute confusional status (Delirium). Delirium was confirmed based on psychiatry consultation, glasgow coma scale (GCS) scoring system, and using the confusion assessment method (CAM). The patient was already diagnosed with Dementia based on conclusive diagnostic criteria $(2,3)$. The final psychiatric assessment, confirmed the diagnosis of delirium superimposed on dementia (DSD). The patient was treated with Gabapentin $300 \mathrm{mg}$ oral tablet once daily. However, features of delirium kept fluctuating during the day, and being exacerbated at night. The resultant insomnia was distressing to the patient, medical staff, and the caregivers.

Topical wound care was done in parallel, and a coexisting cellulitis was excluded via microbiological stud- 
ies. Topical wound (venous ulcer) care, included the use of hydrogen peroxide, povidone iodine, topical Rifampicin, and an occlusive wound dressing that was changed twice daily.

Concerning the DVT management, the patient was immediately advised against movement, to avoid a catastrophic pulmonary embolism (PE). Low molecular weight heparin (LMWH), in the form of Enoxaparin subcutaneous injections, adjusted for both age and weight, was administered for three successive days, followed by an adjusted dose of warfarin tablets for six weeks, and Pentoxifylline $400 \mathrm{mg}$ tablets twice daily. Laboratory investigations of blood coagulation profile (PT, PTT, and INR), RFT, and serum electrolytes, were repeated on a weekly basis. A second doppler study was done after four weeks from the onset of this management protocol. Luckily, the Doppler study confirmed complete resolution of the DVT and the absence of any arterial pathology. However, the hard necrotic area above the medial malleolus, continued to expand over the course of some weeks. Abbade et al. stated that venous leg ulcer is responsible for about $70 \%$ of chronic ulcers of the lower limbs, and recurrence is quite high, ranging from 54 to $78 \%(7,8)$.

Vascular surgery consultation, advised against any sort of surgical debridement of the necrotic tissue, due to the patient's age, elevated renal function test, and her overall immune status. Patient's right leg and foot edema became gradually more evident, with an increasing level of discomfort and pain, despite normal Doppler studies. The patient eventually developed oliguria, with a persistently elevated RFT, and a borderline serum potassium level. As a result, the patient was hospitalized, on the 3rd of March 2016. A biopsy was taken from the necrotic leg ulcer, and it confirmed bacterial colonization. The patient was treated with intravenous Ceftazidime $2 \mathrm{gm}$ three times a day, intravenous Vancomycin $1 \mathrm{gm}$ twice a day, and potassium sparing diuretics. Orthopedic consultation and radiological study of both legs, excluded the presence of osteomyelitis. The patient also underwent a computerized tomography (CT) scan of the head, which revealed no evident structural abnormalities of the brain, which may provide an alternative explanation of her mental status (9).

Patient's hydration level was assessed and accordingly corrected with intravenous fluid replacement, while simultaneously monitoring the daily urine output (UOP). Concerning the delirium episodes, they were frequent, interfering with sleep particularly at night. Food intake was also minimal, for which a nasogastric tube was suggested, but not considered practical, due to the hyperactive episodes of delirium. Total parenteral nutrition was not considered due to patient's elevated renal parameters. Gabapentin was discontinued, and haloperidol $1.5 \mathrm{mg}$ tablet once daily at night, was prescribed. Seitz et al. in his systematic review on the use of antipsychotics in the treatment of Delirium, found that the medications most frequently utilized, are the typical antipsychotic haloperidol, various benzodiazepines, and increasingly, atypical antipsychotics (10). The patient RFT was re-evaluated after four days, and it was fully normal. The leg edema regressed, and the delirium became less frequent and less evident, and the patient was discharged and her GCS at the time of discharge was 15/15. Topical wound care was redefined with topical fusidic acid and an occlusive dressing till complete sloughing occurred. Later, the sloughed and exuding ulcer was dressed with an occlusive hydrocolloid dressing. Physiotherapy of both lower limbs was also instructed. Gradually, patient's food intake returned to normal, and the frequency of Delirium episodes were significantly reduced.

Autologous mesh skin grafting for the ulcer site was planned, under regional anesthesia (nerve block) under cover of LMWH. The rationale for this customized surgical approach was based on the fact that this protocol yielded a success rate of up to $75 \%$ (11).

\section{Discussion}

This case represents a provocative medical problem that required an MDT team of a vascular surgeon, a dermatologist, an internal Medicine specialist, paramedical staff and caregivers. The cornerstone challenge to both the physicians and the caregivers was that the patient was an elderly patient, who developed DSD, in parallel with other medical complications including dehydration and subsequent decline in RFT, insomnia, hyperactivity and aggression as a result of the acute confusional state. Surgical complications also arose including venous ulceration as a consequence of chronic venous insufficiency and venous thrombosis. The venous ulceration was not feasible for classical surgical intervention due to patient's advanced age, impaired renal functions and the overall low level of immunity.

The uncontrolled DSD left the patient in a very distressed state, beyond the capacity of the caregivers and the medical staff. Luckily, the patient responded to an adjusted dose of Haloperidol. Once patient's DSD was under control, the patient started to be more cooperative with caregivers, and willingly accepted her medications, and cooperated with the medical and paramedical staff. The patient's condition was finally stabilized and she was discharged from the hospital, to be scheduled later for surgical intervention, using an autologous mesh split-thickness skin grafting, under regional anesthesia. 


\section{Acknowledgments}

Appreciation and gratitude goes to Dr. Nadia AlGbouri, a consultant psychiatrist at Mediclinic in Dubai, for her critical notes and persistent support. We also acknowledge the valuable feedback comments from Dr. Maha Sulaiman, a consultant psychiatrist and a professor of psychiatry at the faculty of Medicine, University of Baghdad.

\section{Footnotes}

Authors' Contribution: Ahmed Al-Imam, the solitary author of this paper was responsible for study concept and design, acquisition of data, analysis and interpretation of data, drafting of the manuscript, and revision of the manuscript according to the reviewers' comments.

Financial Disclosure: None.

Funding/Support: No external funding.

\section{References}

1. Morandi A, McCurley J, Vasilevskis EE, Fick DM, Bellelli G, Lee P, et al. Tools to detect delirium superimposed on dementia: a systematic review. J Am Geriatr Soc. 2012;60(11):2005-13. doi: 10.1111/j.15325415.2012.04199.x. [PubMed: 23039270].

2. McKhann GM, Knopman DS, Chertkow H, Hyman BT, Jack CJ, Kawas $\mathrm{CH}$, et al. The diagnosis of dementia due to Alzheimer's disease: recommendations from the National Institute on Aging-Alzheimer's Association workgroups on diagnostic guidelines for Alzheimer's disease. Alzheimers Dement. 2011;7(3):263-9. doi:10.1016/j.jalz.2011.03.005. [PubMed: 21514250].
3. Bamford C, Lamont S, Eccles M, Robinson L, May C, Bond J. Disclosing a diagnosis of dementia: a systematic review. Int J Geriatr Psychiatry. 2004;19(2):151-69. doi:10.1002/gps.1050. [PubMed:14758581].

4. Decousus H, Frappe P, Accassat S, Bertoletti L, Buchmuller A, Seffert B, et al. Epidemiology, diagnosis, treatment and management of superficial-vein thrombosis of the legs. Best Pract Res Clin Haematol. 2012;25(3):275-84. doi: 10.1016/j.beha.2012.07.005. [PubMed: 22959544].

5. Baggen VJ, Chung K, Koole K, Sarneel MH, Rutten FH, Hajer GR. Association of varicosities and concomitant deep venous thrombosis in patients with superficial venous thrombosis, a systematic review. Eur J Gen Pract. 2015;21(1):70-6. doi:10.3109/13814788.2014.919629. [PubMed: 24909345].

6. Douma RA, Tan M, Schutgens RE, Bates SM, Perrier A, Legnani C, et al. Using an age-dependent D-dimer cut-off value increases the number of older patients in whom deep vein thrombosis can be safely excluded. Haematologica. 2012;97(10):1507-13. doi: 10.3324/haematol.2011.060657. [PubMed: 22511491].

7. Abbade LP, Lastoria S. Venous ulcer: epidemiology, physiopathology, diagnosis and treatment. Int J Dermatol. 2005;44(6):449-56. doi: 10.1111/j.1365-4632.2004.02456.x. [PubMed: 15941430].

8. Green J, Jester R, McKinley R, Pooler A. The impact of chronic venous leg ulcers: a systematic review. J Wound Care. 2014;23(12):601-12. doi: 10.12968/jowc.2014.23.12.601. [PubMed: 25492276].

9. Soiza RL, Sharma V, Ferguson K, Shenkin SD, Seymour DG, Maclullich AM. Neuroimaging studies of delirium: a systematic review.JPsychosom Res. 2008;65(3):239-48. doi: 10.1016/j.jpsychores.2008.05.021. [PubMed: 18707946].

10. Seitz DP, Gill SS, van Zyl LT. Antipsychotics in the treatment of delirium: a systematic review. J Clin Psychiatry. 2007;68(1):11-21. [PubMed: 17284125].

11. Serra R, Buffone G, de Franciscis A, Mastrangelo D, Vitagliano T, Greco M, et al. Skin grafting followed by low-molecular-weight heparin long-term therapy in chronic venous leg ulcers. Ann Vasc Surg. 2012;26(2):190-7. doi: 10.1016/j.avsg.2011.04.008. [PubMed: 22018697]. 